\title{
Role of Phenology in Host Susceptibility and Within-Plant Spread of Stem Rust During Reproductive Development of Perennial Ryegrass
}

\author{
W. F. Pfender
}

U.S. Department of Agriculture-Agricultural Research Service, National Forage Seed Production Research Center/Oregon State University, Department of Botany and Plant Pathology, 3450 SW Campus Way, Corvallis 97331. Accepted for publication 23 October 2003.

\begin{abstract}
Pfender, W. F. 2004. Role of phenology in host susceptibility and withinplant spread of stem rust during reproductive development of perennial ryegrass. Phytopathology 94:308-316.

Perennial ryegrass (Lolium perenne) was inoculated with urediniospores of Puccinia graminis subsp. graminicola at four stages of reproductive tiller development. All developmental stages, from expansion of the penultimate leaf through anthesis, were equally susceptible to infection measured as number of pustules per total inoculated plant area. However, within each stage from boot through anthesis, there was a negative correlation of susceptibility with age of host tissue, the highest disease severities occurring on expanding or newly expanded leaves or inflorescences. Within-plant spread of the disease from primary lesions

produce an elongated strip of contiguous secondary infections on the inflorescence head and stem, which is a characteristic sign of stem rust. Position and timing of eruption of the secondary pustules on the stem can be explained by assuming that infections occur on the stem slightly distal to the overlying sheath lesion and erupt one latent period later, by which time tiller elongation has moved the stem infection site upward. Repeated infections occur as the stem elongates, producing contiguous lesions. Expansion rate and final lesion area on the stem were correlated with elongation rate of the inflorescence. Microscopic observation of plant tissue sections revealed that the fungus sporulates on the inner surface of the sheath, and germinating urediniospores are attached to the stem surface under the covering sheath starting 1 to $2 \mathrm{~cm}$ distal to the location of the sheath infection site.
\end{abstract} on the flag leaf sheath to the elongating stem of the inflorescence was observed and verified. A single primary infection on the leaf sheath can

Host phenology is an important factor affecting development of many diseases (14). Plant age and the age and position of plant organs can affect susceptibility to infection as well as postinfection development such as lesion growth or sporulation. Populer (14) noted several typical patterns of change in susceptibility with time. Depending on the disease, susceptibility of host tissue may increase with time, decrease with time, or be associated with particular growth stages. Grape berries decrease in susceptibility to powdery mildew with age, showing a marked decrease ("ontogenic resistance") at 3 to 4 weeks postbloom (7), and strawberry leaflets become progressively less susceptible to infection by Phomopsis obscurans during their first 2 weeks of age (11). In contrast, leaves of the grass Coix lacryma-jobi become more susceptible to infection by Bipolaris coicis with age (5). Leaves of snap beans show a peak of susceptibility to Uromyces phaseoli when they are $20 \%$ expanded (21). It is important to distinguish among effects due to aging of a given organ, the position of the organ on the plant, and age of the plant itself. For example, leaf position (from basal to apical) is more influential than age of the leaf in determining susceptibility of potato to late blight (22). A phenomenon known as adult plant resistance is common in rust diseases of wheat. For example, leaf rust susceptibility (measured as latent period, infection frequency, and pustule size) was observed to be higher in seedlings than on young flag leaves in a group of 18 wheat cultivars (3). In some cases, knowledge of the effects of plant phenology on disease processes is required for accurate prediction and efficient management of epidemics.

Corresponding author: W. F. Pfender; E-mail address: pfenderw@onid.orst.edu

Publication no. P-2004-0121-01R

This article is in the public domain and not copyrightable. It may be freely reprinted with customary crediting of the source. The American Phytopathological Society, 2004.
Additional keywords: Graminae, wheat.

When grown to maturity to produce a seed crop, perennial ryegrass (Lolium perenne L.) can be severely damaged by Puccinia graminis subsp. graminicola, causal agent of stem rust. In major seed producing areas, such as New Zealand and the Pacific Northwest of the United States, fungicides typically are applied several times in a growing season to manage the disease. Efforts to develop models for epidemics and management of stem rust are in progress (13), and it is necessary to determine the importance of host phenology in epidemic development. In Oregon, fields of perennial ryegrass for seed production are typically sown in the autumn (September to November). After initial growth of the seedlings, plants are exposed to the cold, short-day conditions required to induce flowering (vernalization) (8). Tillering (Feeke's scale 3 to 5 [10]) continues until April in most cultivars followed by stem extension (Feeke's 6 to 9) that reaches the boot stage (Feeke's 10) by mid-May. The leaves have a lamina (blade) component and a sheath component that forms a hollow cylinder enclosing the younger leaves, stem, and inflorescence as they develop from meristem to mature organ. Stem extension involves elongation of internodes that are initially enveloped in the sheaths of lower (older) leaves. The inflorescence of L. perenne is a spike, with spikelets sessile on the distal half of the inflorescence axis. In Oregon, the inflorescence of most cultivars first emerges from the sheath of the top (flag) leaf (Feeke's 10.1) in mid-May and is completely extended by mid-June. The spikelet-bearing portion of the inflorescence of commonly grown cultivars may be 20 to $25 \mathrm{~cm}$ long, subtended by the basal portion (the peduncle), 15 to $20 \mathrm{~cm}$ long. At maturity, the flag leaf sheath is typically 8 to $12 \mathrm{~cm}$ long, and thus encloses the basal one-quarter to one-third of the inflorescence after complete extension of this $35-$ to $45-\mathrm{cm}$ organ from within the sheath. It is a common observation among growers of perennial ryegrass seed crops that stem rust becomes particularly severe around the time of anthesis, after inflorescences have fully extended. This observation has led to a commonly held 
assumption among growers that the plants are especially susceptible to infection at and immediately after the time of inflorescence extension.

Welty and Barker (23) compared perennial ryegrass seedlings and adult plants for susceptibility to infection by $P$. graminis subsp. graminicola. By evaluating susceptibility of individual seedlings to infection, and then measuring disease on the same individual plants as they matured after being transplanted to the field, they concluded that 8-week-old plants are more susceptible than 14-week-old plants and that some plants identified as susceptible at these young stages are relatively resistant to infection as postvernalization adults (23). However, there are no published reports of relative susceptibility of perennial ryegrass to stem rust infection among postvernalization stages from tillering through seed set. In stem rust of wheat, susceptibility to infection can differ among phenological stages from tillering to heading (19), and the pattern of susceptibility with stage is associated with particular resistance genes.

Plant phenology and tissue age can influence postpenetration events, such as lesion growth, in some diseases (14). There are no published data on this phenomenon in stem rust diseases, but typical pustule development and growth rates have been described for stem rust of wheat. Allen (2) observed that postinfection fungal growth by $P$. graminis subsp. graminis can extend through the leaf lamina to the opposite surface, but remains localized. She (2) noted that the fungus can extend its growth laterally, by means of "stolon-like hyphae," away from the original infection site to form additional pustules that expand the colonized area, at the scale of millimeters. Rowell (17) measured rates of stem rust pustule growth in wheat cultivars and noted that they reached lengths of 1 to $2 \mathrm{~mm}$ over the course of 7 days after inoculation.

The objectives of this study were to determine whether there are differences in susceptibility of perennial ryegrass plants to infection by $P$. graminis subsp. graminicola correlated with tissue age and growth stage during host reproductive growth. In addition, the influence of phenological stage on within-plant spread of the pathogen is described and quantified.

\section{MATERIALS AND METHODS}

Plant and pathogen material. Seeds of perennial ryegrass cv. Morningstar were sown into fine vermiculite on the surface of potting soil (Sunshine Growing Mix; Sun Gro Horticulture Inc., Bellevue, WA) in 4-liter pots in mid-October. Plants were grown for 12 weeks in a greenhouse controlled at $21 \pm 3^{\circ} \mathrm{C}$ (day) and $15 \pm 2{ }^{\circ} \mathrm{C}$ (night) and supplemented with artificial lighting (highpressure sodium lamps, $430 \mathrm{~W}$, Philips Lighting, Somerset, NJ) to provide a 16-h day length. Plants were thinned to three plants per pot and moved outdoors in early January to expose juvenile plants to the cold, short days required for floral induction (8). Throughout the experiments, plants were fertilized every 2 weeks with a nutrient solution containing $303 \mathrm{mg}$ each of $\mathrm{N}$ and $\mathrm{K}_{2} \mathrm{O}$ and $273 \mathrm{mg}$ of $\mathrm{P}_{2} \mathrm{O}_{5}$ in $500 \mathrm{ml}$ of water. Urediniospores of $P$. graminis subsp. graminicola used for inoculum were a bulked collection obtained in June of the prior year from several different cultivars of perennial ryegrass at Hyslop Experiment Farm near Corvallis, OR. Urediniospores were collected with a cyclone sampler and dried overnight at $30 \%$ relative humidity and then stored at $-60^{\circ} \mathrm{C}$ until use. Spores were heat-shocked for $1.5 \mathrm{~min}$ at $43^{\circ} \mathrm{C}$ immediately before use (18).

Susceptibility and plant phenology. For the experiments on plant phenology and susceptibility, plants of different growth stages were inoculated at the same time to avoid the variability due to inoculation procedure that could occur if successive stages were inoculated as they developed. Four different stages were induced by bringing plants from the outdoors into the warm, long-day greenhouse on four different dates. The first time the experiment was done (trial 1), different pots were moved into the greenhouse on each of the following dates: 22 January, 5 and 23 February, and 12 March 2001. At inoculation (22 March 2001), plants that had been moved into the greenhouse on 12 March were at Feeke's stage 7 to 8 (penultimate leaf visible), whereas plants that had been moved on 23 and 5 February and 22 January had tillers at Feeke's stage 10 (boot stage), 10.3 (head emerging), and 10.5 (head fully emerged, anthesis beginning), respectively. When the experiment was repeated in 2002 (trial 2), pots were moved from the outdoors into the greenhouse on 15 February, 8 and $21 \mathrm{March}$, and 1 April to produce the same range of plant stages at inoculation date (25 April 2002). From the time plants were brought in from outdoors until the end of the experiment, pots were kept in shallow trays of water so they could be irrigated without applying water to the leaves.

One tiller on each plant was selected for inoculation, so there were three inoculated tillers per pot. Inoculation was accomplished by spraying an individual tiller with a suspension of urediniospores in Soltrol (Philips Petroleum Co., Bartlesville, OK) a light mineral oil (18). A small hand-held Venturi atomizer (4) was operated with an air pump to spray the spore suspension. The tiller was stretched onto a clean stainless steel counter and held in place with small metal weights, and the spore suspension was sprayed the full length of the exposed tiller by moving the handheld sprayer along the tiller at approximately $100 \mathrm{~cm} / \mathrm{s}$. The stainless steel counter was cleaned with $95 \%$ ethanol before each tiller was inoculated. In trial 1 , the inoculum concentration was $2.4 \mathrm{mg}$ of urediniospores per $\mathrm{ml}$ of Soltrol $\left(1 \times 10^{6}\right.$ spores per $\left.\mathrm{ml}\right)$, and the inoculation procedure delivered 40 spores per $\mathrm{cm}^{2}$ (standard deviation $=13$ ), as determined by counts of spores deposited on glass microscope slides sprayed at the same time as the tillers. In trial 2, the inoculum concentration was $12 \mathrm{mg}$ of urediniospores per ml, delivering 200 spores per $\mathrm{cm}^{2}$. After allowing 1 to $2 \mathrm{~h}$ for oil to evaporate from the leaves, plants were placed overnight $(15 \mathrm{~h})$ in a chamber of plastic sheeting with a light-proof cover to maintain darkness, supplied intermittently with mist (20 min on, $40 \mathrm{~min}$ off), and held at $20^{\circ} \mathrm{C}$. In the morning, the dark cover was removed from the chamber to let in natural light, mist was applied continuously, and the temperature was gradually raised to $25^{\circ} \mathrm{C}$ over the next $4 \mathrm{~h}$. The plants were moved from the mist chamber to the greenhouse bench to dry and kept under the same growing conditions as before inoculation. Air temperature in the greenhouse was recorded every half-hour by means of a temperature logger (HOBO Temp Probe, Onset Computer Corp., Pocasset, MA), and these data were used to calculate latent period duration (12) for the experiments.

The plant surface area inoculated was calculated by measuring blade length and width (at one-third the distance from the base) of each leaf and length and diameter of each stem-associated organ (sheath, inflorescence stem, and head) on the day of inoculation. Leaf blade area was calculated as area $=0.837 \times$ length $\times$ width. This equation was derived from a linear regression analysis relating length $\times$ width to actual area measured with a leaf area meter (Area Meter A100, ADC Plant Science Instrumentation, Hoddesdon, Hertfordshire, England) for 20 leaves of perennial ryegrass spanning the size range of those encountered in the experiments. Area of stem-associated organs was calculated as $1 / 2 \times(\pi \times$ diameter $\times$ length $)$ to account for inoculating only one side of the stem organs. Area of the inflorescence was calculated from length, based on empirical measurements taken with the leaf area meter, as area $=0.183 \times(\text { length })^{1.18}$

Length measurements also were taken at the end of the experiment. Comparison of final length with length at the time of inoculation revealed whether a particular leaf, sheath, stem, or inflorescence had been fully expanded when inoculated. Developmental age of an expanding organ was calculated as the ratio of (length when inoculated)/(final length) and expressed as a proportion ( 0 to 1). The developmental age of each additional (i.e., older) plant organ was expressed as the sum of its proportional size (1.0 for 
fully expanded organs) plus that of all younger organs. Each leaf blade, sheath, inflorescence, and inflorescence stem were considered separate organs in calculating the sum for developmental age. For example, on a plant with no exposed inflorescence, the flag leaf blade $75 \%$ expanded and all lower organs fully expanded, the flag leaf blade was assigned a developmental age of 0.75 , its sheath was 1.75 , the penultimate leaf blade was 2.75 , and its sheath was 3.75 .

The number of pustules per plant organ (each leaf blade, leaf sheath, inflorescence head, and stem) on each inoculated tiller was determined 14 days, or approximately 1.5 latent periods, after inoculation. Pustule number per inoculated plant area was calculated separately for each plant organ based on the area present on the day of inoculation.

For statistical analysis of disease severity as a function of phenological stage and leaf position, the experimental unit was taken to be one pot containing three inoculated tillers. There were six pots per treatment (phenological stage) and four phenological stages. Six pots per stage were left noninoculated as a check on extraneous infection. To test phenological stage as a factor in average susceptibility of plants, a one-way analysis of variance (ANOVA) on whole-plant averages of infection severity (total number of pustules divided by total inoculated area for each pot) was used. A two-way ANOVA (with organ position as the second factor) was not used, because the younger stages did not include all the organs (e.g., flag leaf and inflorescence) present in older stages. For the evaluation of organ position as a factor in susceptibility, a one-way ANOVA was used within each developmental stage to test organ type and position as a factor affecting the number of pustules per inoculated area. For this analysis, the number of pustules per inoculated leaf area for each plant organ was averaged across the three tillers in each pot to produce each replicate value. Data from noninoculated checks were not included in the analysis because no disease was observed on them. Prior to conducting the ANOVA procedures, the data sets were tested and found to be nonnormal, with unequal variances among treatments. Transformation to $\log _{10}$ equalized the variance and rendered the data normal. Therefore, ANOVAs were conducted on log-transformed data. The means were back-transformed for presentation in the Tables. The relationship of developmental age to susceptibility of individual organs was examined by performing a Spearman's rank correlation analysis (which does not require normality) on the nontransformed data set consisting of all paired observations of developmental age and number of pustules per unit plant area for each individual organ of each tiller. All data analyses were performed using SigmaStat software (SPSS, Inc., Chicago).

TABLE 1. Severity of stem rust on perennial ryegrass plants inoculated at different phenological stages with Puccinia graminis subsp. graminicola

\begin{tabular}{lccc}
\hline $\begin{array}{l}\text { Phenological stage } \\
\text { at inoculation }\end{array}$ & $\begin{array}{c}\text { Feeke's } \\
\text { stage }\end{array}$ & Trial $1^{\mathrm{z}}$ & Trial $2 \mathrm{z}^{\mathrm{z}}$ \\
\hline Tiller extending & 7 to 8 & $5.9(0.6-41.7)$ & $46.8(12.8-119.2)$ \\
Head in boot & 10 & $4.1(0.8-11.9)$ & $8.1(4.1-13.8)$ \\
Head emerging & 10.3 & $9.3(1.8-17.2)$ & $24.5(2.6-127.8)$ \\
Head extended & 10.5 & $3.3(0.7-23.6)$ & $13.5(1.4-122.0)$ \\
& & ns $(P=0.31)$ & ns $(P=0.10)$ \\
\hline
\end{tabular}

y Plants were grown outdoors in pots (three plants per pot) and brought into a greenhouse at staggered times during the winter in order to obtain different phenological stages at a single inoculation date for each trial.

${ }^{\mathrm{z}}$ Individual tillers (one per plant) were inoculated by spraying with urediniospores of $P$. graminis subsp. graminicola. After inoculation, plants were exposed to controlled overnight conditions to favor infection and then maintained in a greenhouse for approximately 1.5 latent periods before evaluating. Disease severity for each experimental unit (one pot containing three inoculated tillers) was calculated as the quotient of all pustules divided by total plant area inoculated and is indicated in parentheses. Data normalized to $\log \left[1+\left(\right.\right.$ number of pustules per $\left.\left.10 \mathrm{~cm}^{2}\right)\right]$ for analysis. Backtransformed values (pustules per $10 \mathrm{~cm}^{2}$ ) are shown for the average and range of six pots per treatment.
Within-plant spread. To test the hypothesis that the stem rust pathogen can spread to the elongating tiller stem from a single leaf sheath lesion, individual lesions were induced on the flag leaf sheath of plants in the boot to early head emergence stages of development. Urediniospore inoculum was prepared as described in the previous section, at a concentration of $6 \mathrm{mg}$ of spores per $\mathrm{ml}$ of Soltrol. A single tiller was inoculated on each test plant. A site to be inoculated on the flag leaf sheath was marked with two spots of India ink approximately $0.5 \mathrm{~cm}$ apart along the long axis of the sheath in the upper (distal) half of the sheath. The tiller was stretched out on a clean stainless steel counter with the ink spots facing up. A sheet of plastic-backed absorbent bench paper (Labmat Absorbent Liner; Bel-Art Products, Pequannock, NJ) with a reinforced waterproof hole in the middle (waterproof tape on both sides of the bench paper with a 7-mm-diameter hole punched through all layers) was placed over the tiller, with the hole centered on the area between the two ink spots. The sheet of bench paper was held in place with small weights around the inoculation site, and the spore suspension was briefly sprayed on the inoculation site. The bench paper was removed and the oil was allowed to evaporate from the inoculated spot. Plants were placed for $15 \mathrm{~h}$ in a dark chamber at $17^{\circ} \mathrm{C}$ and supplied intermittently with mist (20 min on, $40 \mathrm{~min}$ off) and then exposed to light and gradual drying for $4 \mathrm{~h}$ as the temperature rose to $26^{\circ} \mathrm{C}$. Twelve plants were inoculated on the flag sheath, and an equal number of plants were used as noninoculated checks. At the same time, 12 additional plants at a later stage of development were inoculated at a single site on the stem of the extended inflorescence to assess rate of lesion elongation for individual infections on the stems. After removing plants from the mist chamber, they were maintained in a greenhouse with day temperatures of $23 \pm 3^{\circ} \mathrm{C}$ and night temperatures of $17 \pm 2^{\circ} \mathrm{C}$. The inoculation procedure resulted in single pustule at the inoculation site on the sheath, although in some cases the observed pustule may have been the result of more than one infection in close proximity to one another. In all cases, the pustule on the sheath was localized to the $7-\mathrm{mm}$ inoculation site at the time it erupted.

To characterize elongation rates and relative positions of the flag sheath and the enclosed inflorescence, daily measurements were made of sheath length, exposed length of inflorescence head and stem, and location and size of erumpent rust pustules on these organs. Time of appearance of primary infections (on the sheath) and secondary infections (on the inflorescence head and stem) was recorded. Rate of lesion elongation was calculated from the length and time measurements. Elongation rate also was calculated for primary infections on inoculated stems. Latent period duration was calculated from air temperature measured at halfhour intervals as previously described (12).

The experiment was conducted twice. The association of sheath lesions with elongated lesions on stems was tested by constructing a contingency table for plants with and without sheath lesions and those with and without elongated stem lesions. The significance of association between presence of sheath lesions and stem lesions was tested using Fisher's exact test. Linear regression analysis was used to relate stem lesion expansion rate to stem elongation rate. One-way analysis of variance was performed to test for differences among expansion rates of primary infections on stems, primary infections on sheaths, and secondary infections on stems. Analyses were conducted using SigmaStat.

On additional plants not used in the inoculation experiment, the position of the elongation zone for the inflorescence was assessed by applying dots of India ink at 1-cm intervals along the inflorescence and its stem starting at the subtending node. The ink was applied with a fine-point pen inserted through the overlying flag leaf sheath. At defined time intervals after marking the plants they were dissected to reveal the ink spots, and the distances between adjacent spots were measured. 
Histological examination of the sheath infection site was made 1 to 3 days after eruption of primary sheath pustules on several additional plants inoculated as described. Segments (6 $\mathrm{mm}$ long) of flag sheath and enclosed stem were excised at the inoculation site and distal to it, after first anchoring the relative positions of sheath and stem by inserting a fine thread through the structures. The segments were fixed in formalin-acetic acid, dehydrated, and embedded in paraffin according to standard procedures (20). Transverse sections were cut at approximately $12 \mu \mathrm{m}$ thickness, mounted on slides, and stained with acid fuchsin in lactophenol (6) for microscopic observation.

\section{RESULTS}

Plant phenology and susceptibility. When plants were inoculated with urediniospores of $P$. graminis subsp. graminicola, the average full-tiller susceptibility to infection (number of pustules divided by plant area inoculated) was not significantly different among the growth stages tested (Table 1). That is, a given level of inoculum produced about the same number of infections per inoculated unit area of the tiller whether plants were in the stem extension stage, boot stage, head emergence stage, or fullyemerged head stage. Within each growth stage, however, there were significant $(P<0.05)$ differences in disease severity (pustules per plant area) among plant organs of different positions on the tiller. Differences occurred in both trials within each of the four phenollogical stages that were tested (Table 2). For tillers at Feeke's scale 7 to 8 , the leaf blades were equally susceptible, whereas some of the lower (older) leaf sheaths were less susceptible. For the other growth stages, plant organs with the highest density of pustules included one or more of the most recently exposed plant organs, although in some cases, older organs also were as severely diseased as young organs. For tillers in the boot stage, the flag leaf sheath was particularly receptive to infection.
When the head was emerging from the boot, the flag leaf sheath and the newly exposed inflorescence were especially susceptible. After the inflorescence had emerged completely and its subtending stem was newly exposed above the ligule of the flag leaf, this stem was significantly more receptive to infection than most other parts of the plant.

The relationship between plant tissue age and susceptibility was examined further by plotting disease severity against developmental age for all the individual observations (Figs. 1 and 2). Observations were first divided into two groups, stem-associated organs (leaf sheaths and inflorescence head and stem) (Fig. 1) and leaf blades (Fig. 2), and disease severity for each individual plant organ was plotted against the developmental age of that organ. The Spearman's rank correlation analysis for each plant organ group showed a significant $(P<0.01)$ negative correlation between age and severity of infection. For stem-associated plant organs (Fig. 1), the highest disease severities occurred on tissue that was still elongating (Spearman's rank correlation coefficients of -0.51 and -0.60 for trials 1 and 2 , respectively). Similarly, the highest severity of disease occurred on young leaves (Spearman's rank correlation coefficients of -0.19 and -0.33 , respectively).

Within-plant spread of disease. Approximately 3 weeks after the inoculation date ( 1 week after the previously described data were collected), elongated rust lesions appeared on the inflorescence head or its subtending stem on many of the tillers that had been inoculated at the boot or head-emerging stages. The lesions appeared much later than most of the pustules, which had erupted about 9 days after inoculation. Furthermore, the late-appearing pustules erupted on inflorescences that had not yet been exposed at the time inoculum was applied. These observations suggested that the elongated diseased areas were the result of secondary spread within the plant, originating from primary infections on leaf sheaths enclosing the emerging inflorescence. It was hy-

TABLE 2. Severity of stem rust on different plant organs of perennial ryegrass after inoculation with urediniospores of Puccinia graminis subsp. graminicola ${ }^{\mathrm{x}}$

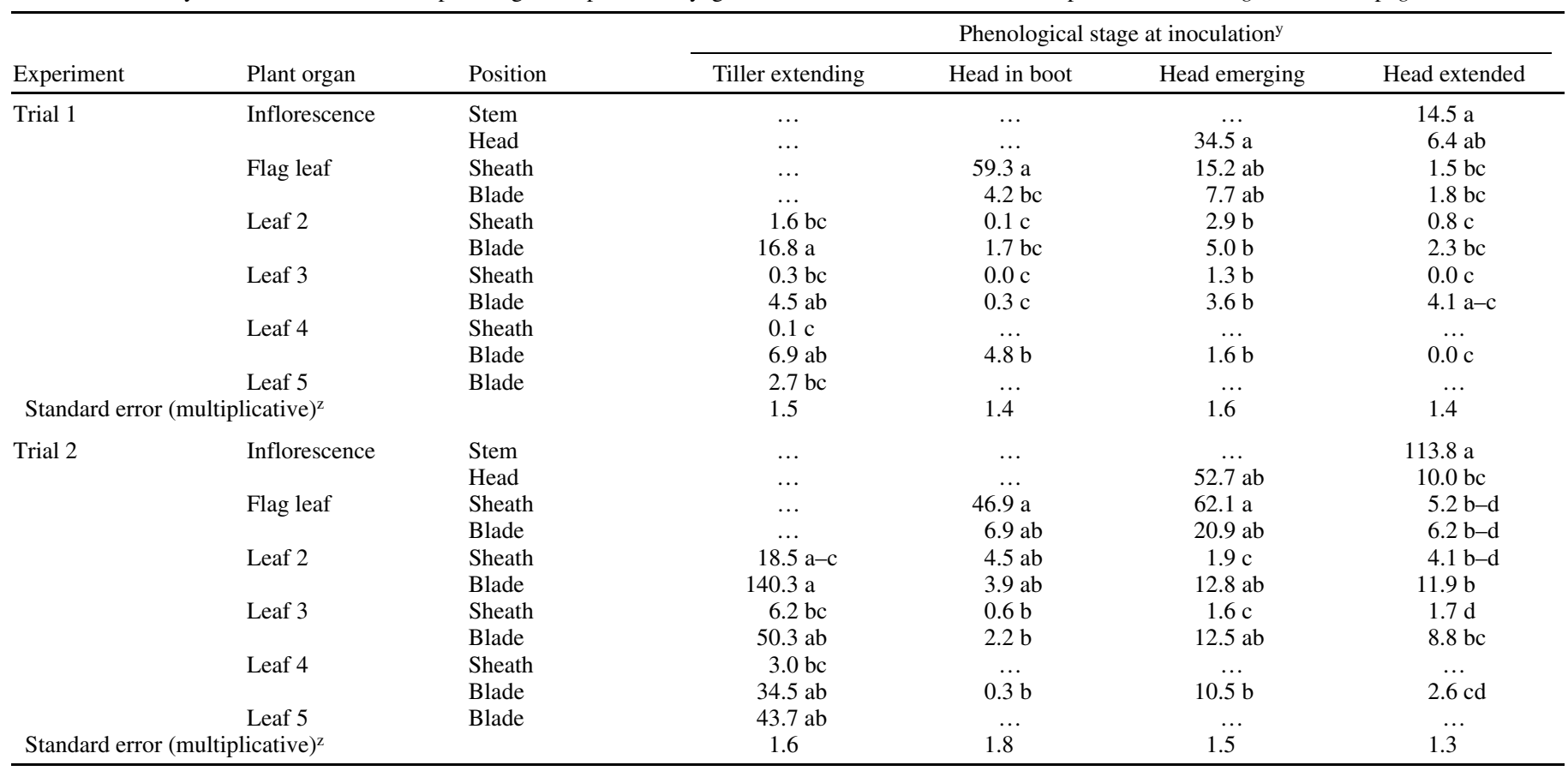

${ }^{\mathrm{x}}$ Disease severity normalized to $\log \left[1+\left(\right.\right.$ number of pustules per $\left.\left.10 \mathrm{~cm}^{2}\right)\right]$ for analysis and back-transformed to pustules per $10 \mathrm{~cm}^{2}$ for presentation. Individual tillers (one per plant) were inoculated by spraying with a suspension of urediniospores in soltrol oil. After inoculation, plants were exposed to controlled overnight conditions to favor infection and maintained in a greenhouse for approximately 1.5 latent periods before evaluation.

y Plants were grown outdoors in pots (three plants per pot) and brought into a greenhouse at staggered times during the winter to obtain different phenological stages at a single inoculation date for each trial. Within a trial, values within a column followed by the same letter do not differ according to mean separation by the Student-Newman-Keuls procedure $(P=0.05)$.

${ }^{\mathrm{z}}$ The back-transformed value for the standard error is multiplicative, rather than additive, because the analysis was performed on log-transformed values. The multiplicative standard error is an indicator of variance expressed as a ratio rather than as an arithmetic difference between means. 
pothesized that primary infections on the flag leaf sheath penetrate its inner surface, where they provide inoculum for multiple, contiguous infections of the enclosed inflorescence as it moves upward past the adaxial surface of the primary infection site on the sheath.

To test the hypothesis of secondary spread on elongating tillers, individual tillers were each inoculated at a single site on the flag leaf sheath. Elongated inflorescence/stem lesions (Fig. 3) later occurred on most of the tillers that had a pustule on the sheath. In the combined data from two trials, there were 24 tillers with a primary lesion on the sheath, and 19 of these tillers (80\%) had elongated, secondary stem lesions. There were no lesions produced on the inflorescences of any tillers that lacked a lesion on the flag leaf sheath (24 tillers). There was thus a clear association (Fisher's exact test $P<0.001$ ) between lesions on the sheath and elongated lesions on the emerging inflorescence.

The rate of lesion elongation for the secondary stem lesions (13.0 and $7.4 \mathrm{~mm} /$ day in the two trials, respectively) (Table 3) was far greater than the elongation rate for primary infections produced by inoculation of single sites on the sheath $(0.3$ to $0.4 \mathrm{~mm} /$ day) or on the emerged stem ( 0.4 to $0.5 \mathrm{~mm} / \mathrm{day})$, indicating that the large size of the secondary lesions was due to some process other than growth of a single lesion. It was hypothesized that the rate of secondary stem lesion elongation is a direct func-
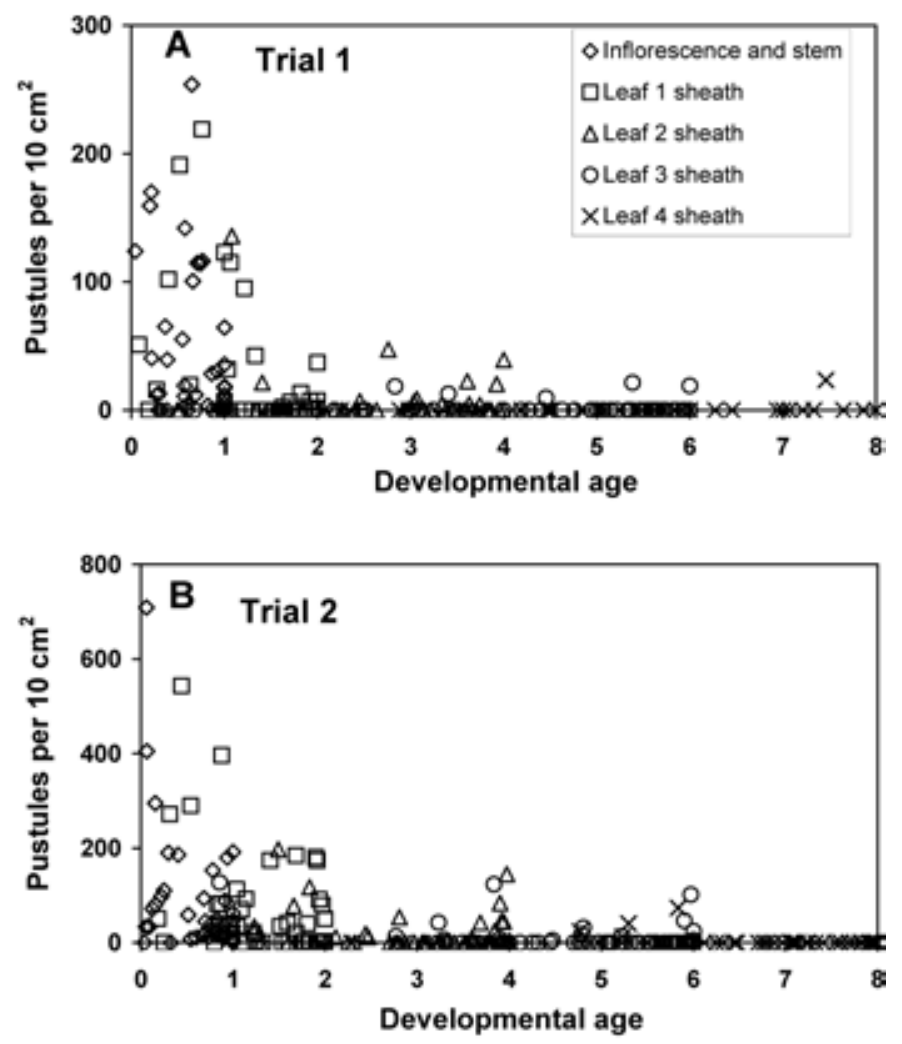

Fig. 1. Relationship between stem rust severity and developmental age of inoculated inflorescences and leaf sheaths of perennial ryegrass plants. Individual observations are shown for two trials (A and B). The flag leaf is labeled leaf 1 ; the penultimate leaf is labeled leaf 2, and so on. Developmental age reflects the relative age of an organ within a plant at the time of inoculation, regardless of its final position on the mature tiller. For the youngest organ present, age is expressed as a proportion ( 0 to 1$)$ of full expansion. The developmental age of each additional (i.e., older) organ is expressed as the sum of its proportional size (1.0 for fully expanded organs) plus that of all younger organs present at time of inoculation. For example, values of $<1.0$ indicate the youngest, partially expanded organ, whereas the oldest organ on the plant has the highest value for developmental age. Spearman's rank correlation analysis showed a significant $(P<0.01)$ negative correlation $(-0.51$ and -0.60 for trials 1 and 2 , respectively) between organ age and disease severity. tion of the rate of stem elongation past the overlying sheath infection. Figure 4A illustrates data from two tillers with respect to expansion of the stem secondary lesion and elongation of the stem that had occurred one latent period prior to stem lesion expansion. The extent and rate of expansion of the secondary lesion is similar to extent and rate of elongation of the stem over the presumed duration of secondary infection for both of these tillers. Regression analysis for combined data from all tillers in the two trials of the experiment indicates a significant relationship between lesion expansion rate and the stem elongation rate one latent period prior to lesion appearance, with slope and intercept not significantly different from 1 and 0 , respectively (Fig. 4B). The final size of secondary stem infections is thus determined by the amount of stem elongation that occurs after the start of spread of infection from the overlying sheath. Under the conditions of these experiments, final lengths of secondary stem infections ranged from 31 to $144 \mathrm{~mm}$ and averaged approximately $80 \mathrm{~mm}$ (Table 3 ).

If infection on the inflorescence occurs directly from the overlying sheath infection, there should be a predictable spatial relationship between the sheath and stem lesion sites at the time of stem infection. This relationship was examined by calculating the relative positions of sheath and stem one latent period prior to pustule eruption of the distal end of the stem (secondary) infection. The latent period duration was calculated as previously de-
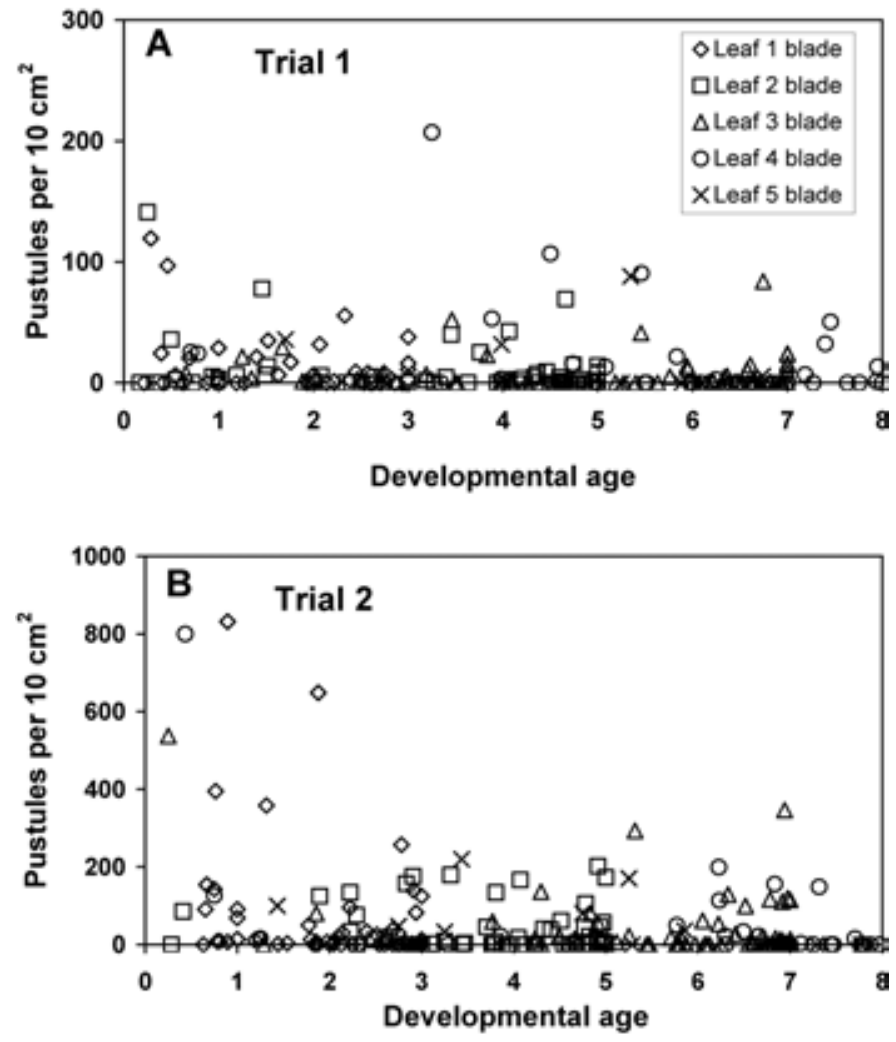

Fig. 2. Relationship between stem rust severity and developmental age of inoculated leaf blades of perennial ryegrass plants. Individual observations are shown for two trials (A and $\mathbf{B}$ ). The flag leaf is labeled leaf 1; the penultimate leaf is labeled leaf 2, and so on. Developmental age reflects the relative age of an organ within a plant at the time of inoculation, regardless of its final position on the mature tiller. For the youngest organ present, age is expressed as a proportion (0 to 1) of full expansion. The developmental age of each additional (i.e., older) organ is expressed as the sum of its proportional size (1.0 for fully expanded organs) plus that of all younger organs present at time of inoculation. For example, values of $<1.0$ indicate the youngest, partially expanded organ, whereas the oldest organ on the plant has the highest value for developmental age. Spearman's rank correlation analysis showed a significant $(P<0.01)$ negative correlation $(-0.19$ and -0.33 for trials 1 and 2 , respectively) between organ age and disease severity. 
scribed (12) using temperature data collected at 30-min intervals during the course of the experiment. The latent period duration was subtracted from the time of first eruption of stem secondary pustules to estimate the time of stem infection for each tiller. The position of the distal point of the eventual stem lesion (centimeter below flower apex) at this estimated infection time was calculated from daily length measurements of sheath and inflorescence and sheath pustule position. Infection was calculated to have occurred when the stem infection site was $7.3 \pm 5.5 \mathrm{~mm}$ above the sheath pustule site in trial 1 and $7.7 \pm 7.1 \mathrm{~mm}$ in trial 2 (means and $95 \%$ confidence interval). From the observed rate of inflorescence extension near the time of estimated stem infection, these distances are equivalent to an average of $13.5 \mathrm{~h}$ after the sheath pustule site and stem infection site are aligned.

Histological examination of the stem and enclosing sheath confirmed that the fungus penetrated the full thickness of the sheath,

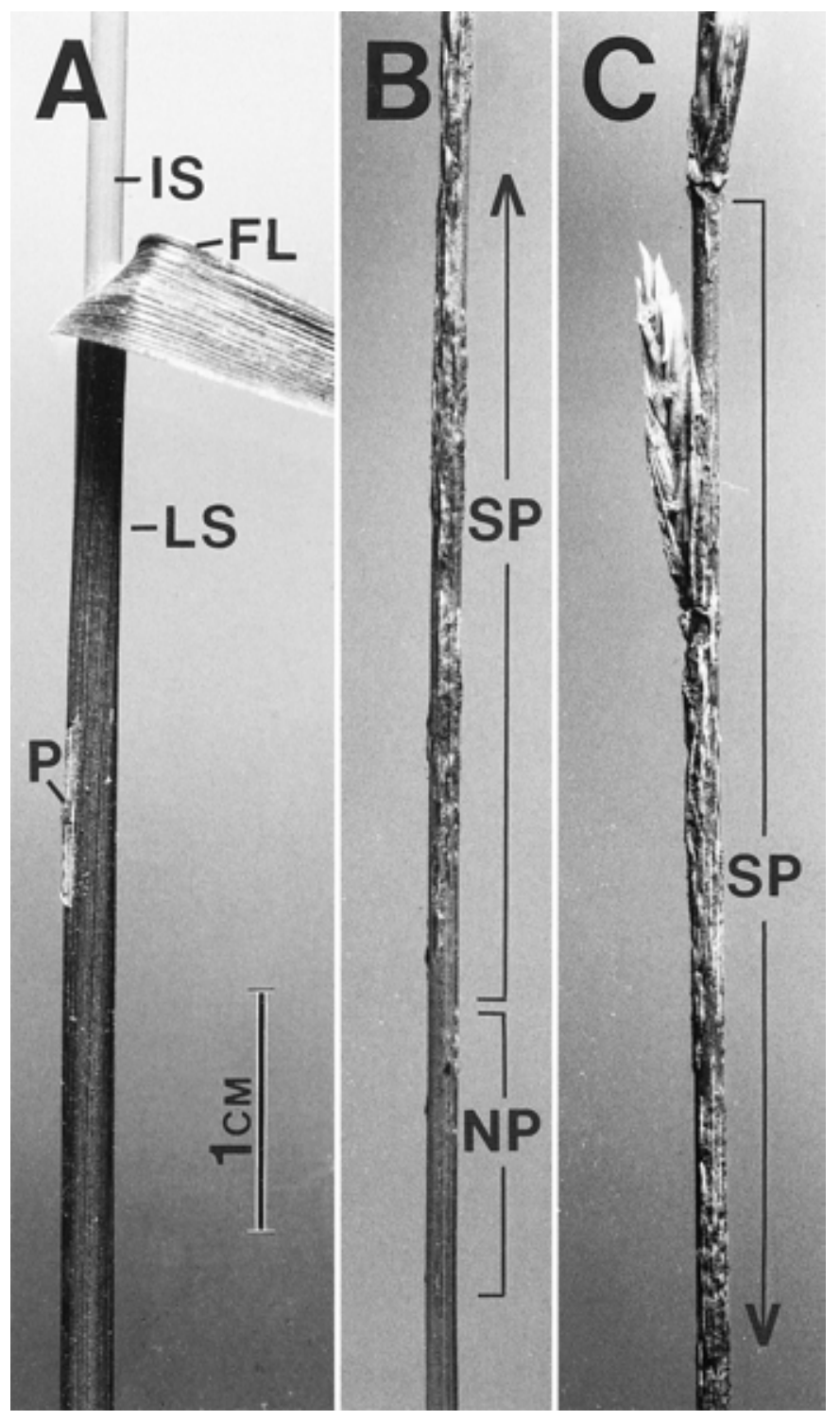

Fig. 3. Perennial ryegrass tiller with pustules of stem rust (caused by Puccinia graminis subsp. graminicola) resulting from a single infection on the flag leaf sheath. Photographs were taken 24 days after sheath was inoculated. A, Pustule (P) resulting from primary infection on sheath (LS) of flag leaf (FL). Sheath was inoculated when inflorescence head was just emerging from sheath. Inflorescence stem (IS) has now completed its extension. B, Portion of the inflorescence stem that is 5 to $10 \mathrm{~cm}$ distal to pustule on leaf sheath. Elongated rust lesion is the result of coalescence of pustules (SP) from secondary infections, the most recent of which are nonerumpent (NP), i.e., just at the end of their latent period. C, Portion of the inflorescence that is 12 to $17 \mathrm{~cm}$ distal to sheath infection. producing sporulating pustules on both the adaxial and abaxial surfaces that erupted at about the same time (Fig. 5A). By 2 days later, urediniospores released from the adaxial pustule had germinated at the surface of the enclosed stem (Fig. 5B), even when

TABLE 3. Size and elongation rates of stem rust pustules resulting from primary or secondary infections on perennial ryegrass tillers

\begin{tabular}{lccccc}
\hline & \multicolumn{2}{c}{$\begin{array}{c}\text { Final lesion } \\
\text { length }(\mathrm{mm})^{\mathrm{z}}\end{array}$} & & \multicolumn{2}{c}{$\begin{array}{c}\text { Lesion elongation } \\
\text { rate }(\mathrm{mm} / \mathrm{day})^{\mathrm{z}}\end{array}$} \\
\cline { 2 - 3 } \cline { 6 - 7 } Infection origin & Trial 1 & Trial 2 & & Trial 1 & Trial 2 \\
\hline Stem primary infection & $8.6 \mathrm{a}$ & $7.0 \mathrm{a}$ & & $0.4 \mathrm{a}$ & $0.3 \mathrm{a}$ \\
Sheath primary infection & $7.5 \mathrm{a}$ & $5.1 \mathrm{a}$ & & $0.4 \mathrm{a}$ & $0.5 \mathrm{a}$ \\
Stem secondary infection & $80.6 \mathrm{~b}$ & $80.4 \mathrm{~b}$ & & $13.0 \mathrm{~b}$ & $7.4 \mathrm{~b}$ \\
Standard error & 6.2 & 7.7 & & 0.6 & 0.7 \\
\hline
\end{tabular}

y Urediniospores of Puccinia graminis subsp. graminicola were applied at a single location to each grass tiller, either on the sheath of the flag leaf at early head emergence or on the stem of the inflorescence after head elongation. A primary infection is the pustule that resulted at the inoculation site. Lesions on the elongating inflorescence stem from secondary infections began to appear one latent period after sheath pustules had erupted and resulted from inoculum produced on the adaxial surface of the inoculated leaf sheath at the primary infection site.

${ }^{\mathrm{z}}$ Values within a column followed by the same letter do not differ $(P<0.01)$ according to the Student-Newman-Keuls test.
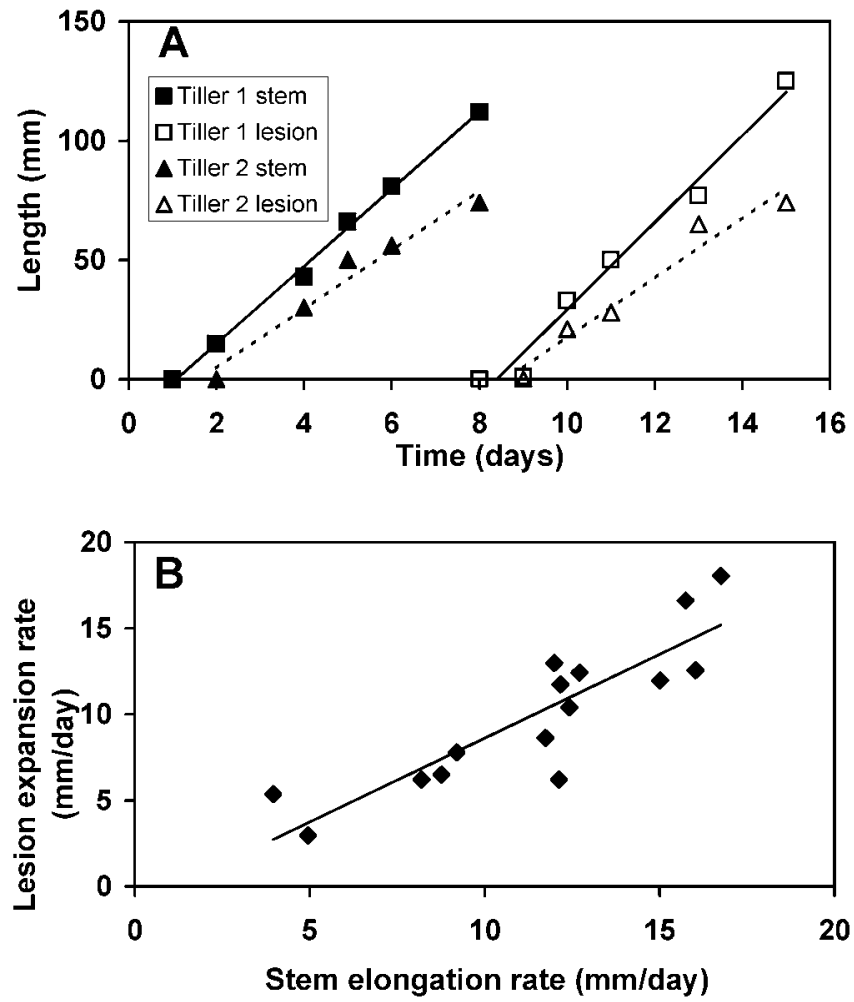

Fig. 4. Comparison of expansion rates of stem lesions caused by Puccinia graminis subsp. graminicola and elongation rates of stems of perennial ryegrass. Tillers were inoculated at a single location on the flag leaf sheath with urediniospores of the pathogen. Length of the inflorescence stem was measured daily, and the calculated stem elongation rate (millimeters per day) was compared with the expansion rate of secondary lesions that erupted on the inflorescence stem one latent period later. A, Data from two tillers, showing that the rate of increase in inflorescence stem length is similar to the rate of lesion expansion one latent period (approximately 7 days under these conditions) later. Elongation rates (slopes) for stem and lesion are 16.2 and $18.2 \mathrm{~mm} /$ day, respectively, for tiller 1 . The respective rates for tiller 2 are 12.4 and $12.6 \mathrm{~mm} /$ day. B, Correlation of lesion expansion rate with prior stem elongation rate. Stem elongation rate was measured at the presumed time of infection (one latent period before lesion expansion measurements). Data are for all tillers in both trials. Regression equation is lesion rate $=$ $[0.98 \times($ stem rate $)]-1.1, R^{2}=0.76$. The slope is not significantly $(P>0.05)$ different from 1 , and intercept is not significantly different from 0 . 
they were present at high density (Fig. 5C). From the germinated urediniospores, the fungus penetrated the enclosed stem (Fig. 5D).

Measurements to determine the position of the zone of elongation for the inflorescence revealed that, near the time of head emergence, elongation occurs only in the basal 2 to $3 \mathrm{~cm}$ of the inflorescence stem. Averaged over 10 tillers, this elongation zone represented the basal $16 \%$ of the inflorescence, covered by the basal $22 \%$ of the flag sheath at the time of sheath inoculation (head emergence stage). Sheath inoculations in these experiments were done on the distal half of the sheaths and therefore they were not near the zone of stem elongation.

\section{DISCUSSION}

The pattern of disease increase over time during an epidemic can be affected by changes in susceptibility with plant age (14) if susceptibility is either limited or exceptionally high during a restricted portion of the host's development. Field observation of markedly increased stem rust severity on perennial ryegrass near the time of anthesis (personal observations) suggests increased susceptibility to the disease at this stage of the plant's development. However, Welty and Barker (23) previously showed that individual perennial ryegrass plants are progressively less likely to be susceptible as they mature from 8-week-old seedlings through 14-week-old plants to postvernalization adults. The results reported here show no significant difference in susceptibility (number of infections per inoculated plant area) among postvernalization stages from late tillering (prior to flag leaf emergence) to full extension of the inflorescence. The similarity in number of infections per unit plant area indicates that there would be no effect on overall epidemic progress resulting from phenology-related susceptibility changes during tiller extension. It should be noted that, in these experiments, the number of infections per unit plant area was taken as a measure of susceptibility. Additional experiments are required to determine whether postinfection phenomena such as lesion growth, spore production, or latent period might be influenced by plant age.

There was a high degree of variability in disease levels in these experiments, likely traceable in part to genetic variability in the host population. Perennial ryegrass has an outcrossing mating system with inbreeding depression and therefore has a high degree of genetic heterogeneity among plants. The experimental data represent the effect averaged over individual plants, and if there is genetic variation for susceptibility to rust infection, the experimental error variance will include the expression of this variability. Because epidemic development is a population phe-

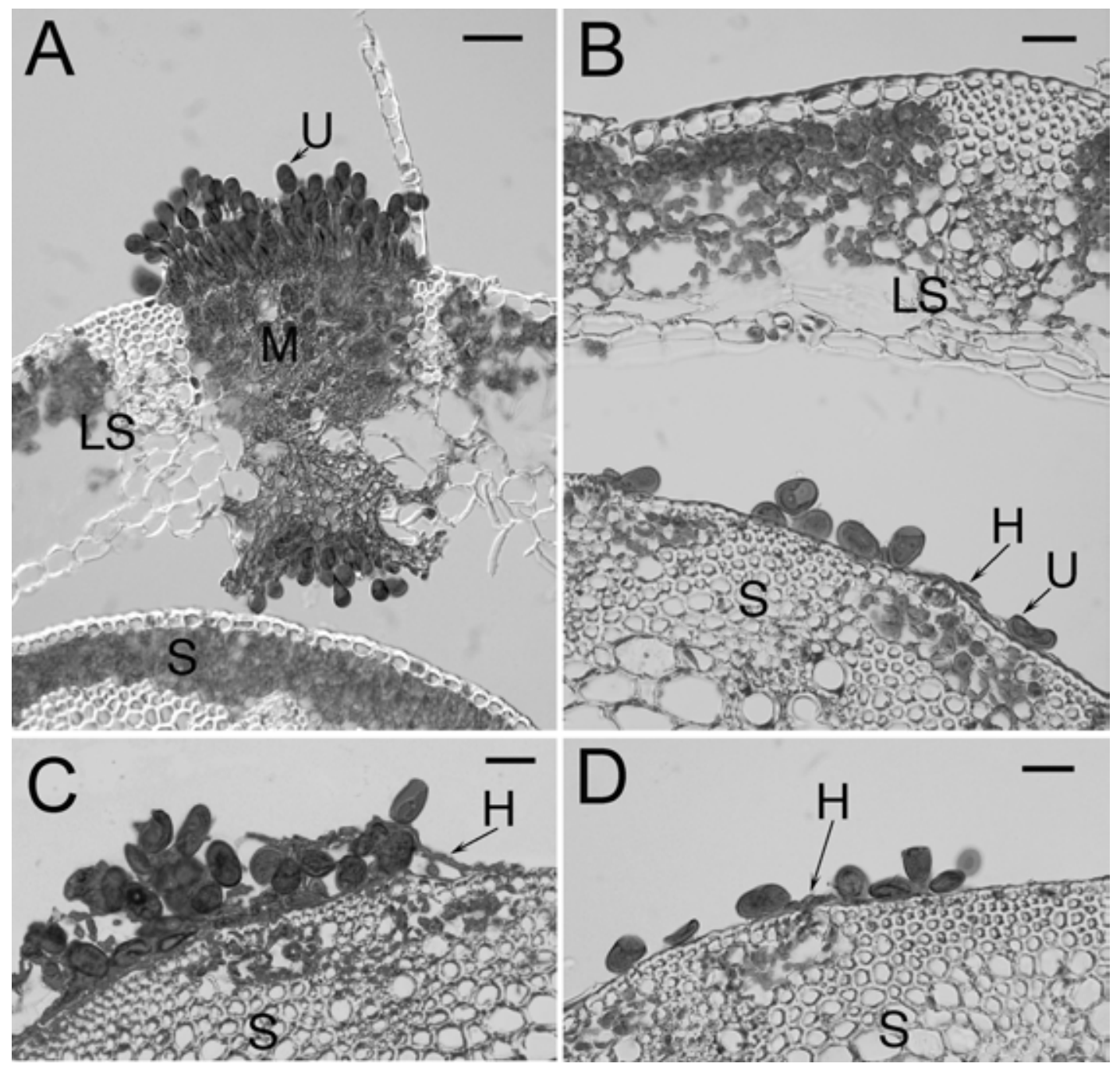

Fig. 5. Cross sections of perennial ryegrass tillers inoculated with Puccinia graminis subsp. graminicola at a single site on the abaxial surface of the leaf sheath. A, Section at the initial inoculation site approximately 1 day after eruption of pustule on abaxial surface of leaf sheath (LS). Fungal mycelium (M) has extended to adaxial surface of leaf sheath, where it produces urediniospores (U) in close proximity to the tiller stem (S) enclosed by the sheath. B to D, Sections 1.5 to $2.0 \mathrm{~cm}$ distal to the sheath infection site taken 3 days after eruption of sheath pustule. The tiller stem is still enclosed within the sheath at this location. B, Urediniospores $(\mathrm{U})$ are attached to the surface of the tiller stem $(\mathrm{S})$, and hyphae $(\mathrm{H})$ from germinating urediniospores are visible. $\mathbf{C}$, Numerous hyphae have emerged from the closely packed urediniospores on the stem surface. D, Hyphae from germinating urediniospores are positioned to penetrate the stem. Scale bars A, $50 \mu \mathrm{m}$ and $\mathbf{B}$ to $\mathrm{D}, 25 \mu \mathrm{m}$. 
nomenon, inclusion of this variability is appropriate in assessing whether there is, on average, a phenological effect on susceptibility that could be translated to a change in disease development within a stand of naturally heterogeneous perennial ryegrass. The results suggest that there is no such effect.

Although phenological stage was not a significant factor in average whole-plant disease susceptibility, individual plant organs within a given plant became less susceptible with age. The highest severity of infection was observed on plant organs that were expanding, and infection severity overall decreased with increasing age of the organs. So, while different plant stages were not differently susceptible to infection, the distribution within each plant was concentrated on expanding or newly expanded organs. This pattern of decreasing susceptibility with tissue age is similar to that seen in some other diseases $(7,11,21)$, although the phenomenon of peak susceptibility of partially expanded leaves observed in bean rust (21) was not observed in the perennial ryegrass stem rust data. In contrast, disease severity was observed to increase with tissue age in some other cases, including diseases caused by either necrotrophic (5) or biotrophic (9) pathogens.

The research reported here relates to a single cultivar, so the effect of phenological stage on susceptibility may not be generalizable to all cultivars. In wheat, individual resistance genes are known to condition adult plant resistance or to decrease susceptibility of leaf sheaths compared with leaf blades (19). These genes can be detected in comparisons of genetically highly homozygous and homogeneous wheat cultivars. Perennial ryegrass occurs as a heterogeneous population, and the existence or role of individual major resistance genes for stem rust in this crop is unknown (16). Therefore, it is likely that the observed lack of difference in average susceptibility at different phenological stages and the observed greater susceptibility of newly developing host tissue are not due to particular major genes unique to this heterogeneous cultivar, but instead are general features of the pathosystem. However, observations on additional cultivars are needed to verify that these are general features.

Whereas the effect of plant and tissue age on susceptibility has only limited bearing on stem rust epidemic development, the impact of phenological stage on within-plant spread is far more important. The adaptation of the pathogen to the morphology and growth dynamics of the host permits a single primary infection on a leaf sheath to be multiplied manyfold through secondary infections on the enclosed, elongating inflorescence or stem. In addition, the newly expanded sheath and enclosed inflorescence are both young tissue and therefore particularly susceptible to infection. Epidemiologically, the result is a very rapid increase in disease severity (and consequently, additional inoculum) starting about one latent period after head emergence, if infections have occurred on the flag leaf sheath. This increase is independent of airborne inoculum and, perhaps to some degree, weather conditions. The consequent surge in disease severity could therefore not be explained without reference to the within-plant spread phenomenon.

Although this study was performed on the flag leaf sheath and enclosed inflorescence, it is likely that the same process occurs on lower sheaths and the structures enclosed there as well. The requirement for within-plant spread to occur at any internode is that the enclosed structures continue to elongate one latent period after the infection occurs on the outer sheath surface, at which time inoculum becomes available on the inner sheath face. The resultant infection process, repeated daily, can produce contiguous lesions along the length of any stem enclosed by an infected sheath, as the stem is pushed up from the elongation zone below. Given the great potential for secondary lesion elongation, within-plant spread may be the predominant mode of stem rust epidemic increase during tiller extension and early flowering in perennial ryegrass. By combining modeling of latent period (12) and plant extension rates, the rate of secondary disease increase within a tiller should be predictable. Because the inoculations reported here were done distal to the zone of elongation at the base of the inflorescence, the estimates of lesion elongation rate were predictable directly from stem extension rate, without the added complication of possible additional lesion elongation due to expansion of host cells at the infection site.

The presence of extensive elongated areas of contiguous rust lesions on the inflorescence and stem of perennial ryegrass is a common observation, but the process by which the phenomenon develops was previously unknown and implicitly assumed to be the result of numerous individual stem infections arising from airborne spores. Elongated stem lesions are typical of stem rust in wheat as well. It is well known that the wheat stem rust fungus can penetrate through a leaf blade and produce sporulating pustules on both surfaces $(2,17)$, and elongated lesions on the inflorescence stem can be produced artificially by injecting a spore suspension into the boot of the developing wheat tiller (15). However, the fact that these elongated lesion areas can derive from single, naturally occurring sheath lesions has not been reported in the literature on wheat stem rust. It seems likely that this phenomenon and its resultant characteristic symptom is a general feature of stem rust diseases in the family Graminae, and is probably very important to pathogen epidemiology and physiology. This feature results from the ability of the pathogen to penetrate and sporulate on the inner surfaces of sheaths, thereby applying inoculum to an extensive length of particularly susceptible tissue as it elongates past the inoculation point. Spores on the stem surface germinate readily after release from the adaxial leaf sheath pustule, despite being crowded. As noted by Allen (1), endogenous inhibitors that prevent germination of crowded urediniospores in some conditions can be overcome by germination stimulators in environments that are advantageous for germination.

The phenomenon of within-plant spread has important implications for disease management. The ability of fungicides to affect spore production at the inner face of the sheath, or to affect spore germination or penetration at the stem surface under the sheath, is critical to their effectiveness. Also, it may be possible to select for host genetic resistance based on a plant's ability to restrict sporulation of $P$. graminis subsp. graminicola on the adaxial surface of the sheath.

\section{ACKNOWLEDGMENTS}

I thank S. Seguin for excellent technical assistance in this research.

\section{LITERATURE CITED}

1. Allen, P. J. 1955. The role of a self-inhibitor in the germination of rust uredospores. Phytopathology 45:259-266.

2. Allen, R. F. 1923. Cytological studies of infection of Baart, Kanred, and Mindum wheats by Puccinia graminis tritici forms III and XIX. J. Agric. Res. 26:571-604.

3. Broers, L. H. M. 1989. Influence of development stage and host genotype on three components of partial resistance to leaf rust in spring wheat. Euphytica 44:187-195.

4. Browder, L. E. 1971. Pathogenic specialization in cereal rust fungi, especially Puccinia recondita f. sp. tritici: Concepts, methods of study, and application. U.S. Dep. Agric. Tech. Bull. 1432.

5. Chang, S. W., and Hwang, B. K. 2003. Effects of plant age, leaf position, inoculum density, and wetness period on Bipolaris coicis infection in adlays of differing resistance. Plant Dis. 87:821-826.

6. Clark, G. 1981. Staining Procedures. 4th ed. Williams \& Wilkins, London.

7. Gadoury, D. M., Seem, R. C., Ficke, A., and Wilcox, W. F. 2003. Ontogenic resistance to powdery mildew in grape berries. Phytopathology 93:547-555.

8. Heide, O. M. 1994. Control of flowering and reproduction in temperate grasses. New Phytol. 128:347-362.

9. Jones, I. T., and Hayes, J. S. 1971. The effect of sowing date on adult plant resistance to Erysiphe graminis f. sp. avenae in oats. Ann. Appl. Biol. 68:31-39.

10. Large, E. C. 1954. Growth stages in cereals, illustration of the Feekes scale. Plant Pathol. 3:128-129. 
11. Nita, M., Ellis, M. A., and Madden, L. V. 2003. Effects of temperature, wetness duration, and leaflet age on infection of strawberry foliage by Phomopsis obscurans. Plant Dis. 87:579-590.

12. Pfender, W. F. 2001. A temperature-based model for latent-period duration in stem rust of perennial ryegrass and tall fescue. Phytopathology 91:111-116.

13. Pfender, W. F. 2003. Prediction of stem rust infection favorability, by means of degree-hour wetness duration, for perennial ryegrass seed crops. Phytopathology 93:467-477.

14. Populer, C. 1978. Changes in host susceptibility with time. Pages 239261 in: Plant Pathology: An Advanced Treatise. J. G. Horsfall and E. C. Cowling, eds. Academic Press, New York.

15. Roelfs, A. P., Singh, R. P., and Saari, E. E. 1986. Rust Diseases of Wheat. CIMMYT, Mexico, D.F

16. Rose-Fricker, C. A., Meyer, W. A., and Kronstad, W. E. 1986. Inheritance of resistance to stem rust (Puccinia graminis subsp. graminicola) in six perennial ryegrass (Lolium perenne) crosses. Plant Dis. 70:678-681.

17. Rowell, J. B. 1981. Relation of post penetration events in Idaed 59 wheat seedlings to low receptivity to infection by Puccinia graminis f. sp. tritici. Phytopathology 71:732-736.
18. Rowell, J. B. 1984. Controlled infection by Puccinia graminis f. sp. tritici under artificial conditions. Pages 291-329 in: The Cereal Rusts, vol. 1. Origins, Specificity, Structure, and Physiology. W. R. Bushnell and A. P. Roelfs, eds. Academic Press, Orlando, FL.

19. Rowell, J. B., and McVey, D. V. 1979. Method for field evaluation of wheats for low receptivity to infection by Puccinia graminis f. sp. tritici. Phytopathology 69:405-409.

20. Ruzin, S. E. 1999. Plant Microtechnique and Microscopy. Oxford University Press, New York.

21. Schein, R. D. 1965. Age-correlated changes in susceptibility of bean leaves to Uromyces phaseoli and tobacco mosaic virus. Phytopathology 55:454-457.

22. Visker, M. H. P. W., Keizer, L. C. P., Budding, D. J., Van Loon, L. C., Colon, L. T., and Stuik, P. C. 2003. Leaf position prevails over plant age and leaf age in reflecting resistance to late blight in potato. Phytopathology 93:666-674.

23. Welty, R. E., and Barker, R. E. 1992. Evaluation of resistance to stem rust in perennial ryegrass grown in controlled and field conditions. Plant Dis. 76:637-641. 